\title{
IMPORTANCE OF TIDES FOR PERIASTRON PRECESSION IN ECCENTRIC NEUTRON STAR-WHITE DWARF BINARIES
}

\author{
N. SRavan ${ }^{1}$, F. VAlsecchi ${ }^{1}$, V. Kalogera ${ }^{1}$, and L. G. Althaus ${ }^{2}$ \\ ${ }^{1}$ Center for Interdisciplinary Exploration and Research in Astrophysics (CIERA), and Department of Physics and Astronomy, \\ Northwestern University, 2145 Sheridan Road, Evanston, IL 60208, USA; niharika.sravan@ gmail.com \\ ${ }^{2}$ Grupo de Evolución Estelar y Pulsaciones, Facultad de Ciencias Astronómicas y Geofísicas, Universidad Nacional de La Plata, \\ Argentina Instituto de Astrofísica La Plata, CONICET-UNLP, Paseo del Bosque s/n, (1900) La Plata, Argentina \\ Received 2013 December 23; accepted 2014 July 15; published 2014 August 26
}

\begin{abstract}
Although not nearly as numerous as binaries with two white dwarfs, eccentric neutron star-white dwarf (NS-WD) binaries are important gravitational-wave $(\mathrm{GW})$ sources for the next generation of space-based detectors sensitive to low frequency waves. Here we investigate periastron precession in these sources as a result of general relativistic, tidal, and rotational effects; such precession is expected to be detectable for at least some of the detected binaries of this type. Currently, two eccentric NS-WD binaries are known in the galactic field, PSR J1141-6545 and PSR B2303+46, both of which have orbits too wide to be relevant in their current state to GW observations. However, population synthesis studies predict the existence of a significant Galactic population of such systems. Though small in most of these systems, we find that tidally induced periastron precession becomes important when tides contribute to more than $3 \%$ of the total precession rate. For these systems, accounting for tides when analyzing periastron precession rate measurements can improve estimates of the inferred WD component mass and, in some cases, will prevent us from misclassifying the object. However, such systems are rare, due to rapid orbital decay. To aid the inclusion of tidal effects when using periastron precession as a mass measurement tool, we derive a function that relates the WD radius and periastron precession constant to the WD mass.
\end{abstract}

Key words: binaries: general - gravitational waves - pulsars: individual (PSR B2303+46, PSR J1141-6545) stars: neutron - white dwarfs

Online-only material: color figures

\section{INTRODUCTION}

Future, space-based gravitational-wave (GW) observations will target Galactic binaries in tight orbits as primary sources of continuous emission (e.g., LISA; Danzmann \& The LISA Study Team 1996; Hughes 2006, and eLISA/NGO, AmaroSeoane et al. 2012). Among those expected to be detected at relatively high signal-to-noise ratio are some eccentric binaries, whose periastron precession will leave an imprint on the GW signal (Willems et al. 2008). In this paper our focus lies on the population of eccentric neutron star-white dwarf binaries (NS-WD). Two such systems are currently observed as radio pulsars orbiting WDs.

Periastron precession in eccentric binaries is caused by a combination of tidal, rotational, and general relativistic (GR) effects. Tides and rotation produce distortions in the binary components, which perturb the stellar gravitational potential from its pure Newtonian form, and the orbit from its Keplerian form, driving precession. Both contributions depend on the internal mass distributions (through the so-called periastron precession constant $k_{2}$ ), masses, and radii of the components, along with the orbital period and eccentricity. The GR contribution, on the other hand, depends solely on the total mass of the system and its orbital elements. Periastron precession is detectable through its imprint on the GWs emitted by these sources. In the absence of periastron precession, the GW radiation is emitted at multiples, $n$, of the orbital frequency, $v_{\text {orb }}$. Periastron precession causes each of these harmonics to split into triplets with frequencies $n v_{\text {orb }} \pm \dot{\gamma} / \pi$ and $n v_{\text {orb }}$, where $\dot{\gamma}$ is the periastron precession rate (e.g., Willems et al. 2008).
Seto (2001) suggests that, if the orbital elements are known, one can use $\dot{\gamma}$ to extract the total mass of low frequency eccentric binaries, assuming that GR effects dominate the periastron precession rate. However, Willems et al. (2008) use polytropic models to investigate periastron precession in eccentric double white dwarf (DWD) binaries and demonstrate that the tidal and rotational distortions of the WD components can significantly affect such a precession in short-period binaries. This implies that ignoring the tidal and rotational contributions, when interpreting periastron precession measurements from these binaries, could lead to an overestimate of the total system mass extracted. Furthermore, they anticipate that accounting for all three contributions would entail degeneracies, given the dependency of $\dot{\gamma}$ on the internal structure $\left(k_{2}\right)$ and radius of both components. Valsecchi et al. (2011) use detailed WD models to study periastron precession in eccentric DWDs and demonstrate that the components' masses could be overestimated by orders of magnitude if tides are not properly taken into account. They also show that a correlation exists between $k_{2} R^{5}$ and the WD mass that allows the use of the periastron precession rate to place constraints on some combination of the components' masses ${ }^{3}$ at any orbital frequency.

Unlike DWDs, where both components contribute to periastron precession, NS-WD binaries are a much cleaner probe

\footnotetext{
3 If GR is the dominant mechanism driving periastron precession, the total system mass can be determined. Instead, if tides are the dominant mechanism, constraints can be placed on $\left(M_{1}+M_{2}\right)^{-5 / 3}\left[k_{1} R_{1}^{5}\left(M_{2} / M_{1}\right)+k_{2} R_{2}^{5}\left(M_{1} / M_{2}\right)\right]$, where the term $k_{i} R_{i}^{5}$ for each WD component $(1,2)$ is a function of its mass according to Equation (7) in Valsecchi et al. (2011).
} 
of WD physics. In such systems, the periastron precession rate carries the sole signature of the WD because the tidal and rotational distortions of the NS contribute negligibly. Furthermore, the theoretically predicted formation rate of galactic eccentric NS-WD binaries is between 10-10,000 times the expected formation rates of eccentric DWDs (e.g., Kalogera et al. 2004, and references therein; Willems et al. 2007). Additionally, because pulsar-timing measurements could yield an independent measurement of the components' masses, these systems may be used to test our predictions and models from analyzing periastron precession rates.

Two eccentric NS-WD binaries, PSR J1141-6545 (Kaspi et al. 2000) and PSR B2303+46 (van Kerkwijk \& Kulkarni 1999), have been discovered in the Milky Way. These binaries have lent support to the existence of a non-traditional formation mechanism for NS-WD binaries (Portgies Zwart \& Yungelson 1999; Tauris \& Sennels 2000; Nelemans et al. 2001; Brown et al. 2001; Davies et al. 2002). The traditional mechanism leads to circular binaries - the more massive primary evolves faster to become an NS, followed by a mass transfer phase from the secondary (WD progenitor), which circularizes any eccentricity introduced by the supernova mechanism that led to the formation of the NS. However, if the progenitors are both massive enough to evolve into massive WDs, the following scenario may unfold. The primary star evolves into a WD after a phase of mass transfer to the secondary. If the secondary acquires enough mass to evolve into an NS, a common envelope forms. During the common envelope phase, the NS progenitor loses its envelope, leaving behind a naked He core in a tight orbit with the WD companion. Subsequently, asymmetries in the NS formation process kick the resulting NS-WD into an eccentric orbit. Apart from this theory, several other evolutionary mechanisms involving multiple mass transfer and common envelope phases have also been proposed (Church et al. (2006)). However, all such mechanisms require an NS-forming supernova explosion as the final step to impart eccentricity to the orbit.

Following Valsecchi et al. (2011), our goal is to investigate the importance of WD tides in driving periastron precession in eccentric NS-WD binaries in order to facilitate an accurate mass interpretation from periastron precession rate measurements. In what follows we also take into account the contribution to periastron precession due to rotation. However, similar to Valsecchi et al. (2011), we find that periastron precession is primarily tidally induced at frequencies were GR is no longer important.

The plan of the paper is as follows. In Section 2 we outline the equations governing the tidal, rotational, and GR contributions to periastron precession. In Section 3 we analyze the importance of tides in driving periastron precession in the observed eccentric NS-WD binary systems. In Section 4 we investigate periastron precession in the population of the eccentric NS-WD binaries in the Milky Way as predicted by population synthesis studies to understand the role of tides. We conclude in Section 5.

\section{PHYSICAL PROCESSES DRIVING PERIASTRON PRECESSION}

We consider an eccentric NS-WD binary system containing an NS of mass $M_{\mathrm{NS}}$, and a WD of mass $M_{\mathrm{WD}}$, radius $R_{\mathrm{WD}}$, and uniformly rotating with angular velocity $\Omega_{\mathrm{WD}}$. We take the NS to be a point mass. We assume that the axis of rotation of the WD is perpendicular to the orbital plane. Let $P$ be the period of the orbit, $a$ the semi-major axis, and $e$ the orbital eccentricity. For simplicity, we take tides to be quasi-static (the regime where the orbital and rotational periods are long compared to the free oscillation modes of the stars; Cowling 1938; Sterne 1939; Smeyers \& Willems 2001), but we note that investigations targeting periastron precession in non-degenerate stars show that the effects of dynamic tides become more significant as the orbital and/or rotational period, and eccentricity increase (Smeyers \& Willems 2001; Willems \& Claret 2002, 2005). We also note that whereas Willems et al. (2010) demonstrate that GR dominates over quasi-static tides in driving the evolution of the orbital separation and eccentricity in NS-WD binaries, recent investigations targeting dynamic tides in binaries hosting a WD and another compact object find that they can significantly speed up the orbital and spin evolution (Burkart et al. 2013; Fuller \& Lai 2011). The contribution to the periastron precession rate, $\dot{\gamma}$, due to quadrupole tides raised in the WD is (Sterne 1939)

$$
\dot{\gamma}_{\mathrm{Tid}, \mathrm{WD}}=\frac{30 \pi}{P}\left(\frac{R_{\mathrm{WD}}}{a}\right)^{5} \frac{M_{\mathrm{NS}}}{M_{\mathrm{WD}}} \frac{1+\frac{3}{2} e^{2}+\frac{1}{8} e^{4}}{\left(1-e^{2}\right)^{5}} k_{2} .
$$

Here, $k_{2}$, also known as the quadrupolar periastron precession constant, is a measure of the WD's central concentration and is given by

$$
2 k_{2}=\frac{\xi_{\mathrm{WD}, T}^{*}\left(R_{\mathrm{WD}}\right)}{R_{\mathrm{WD}}}-1 \text {. }
$$

Here $\xi_{\mathrm{WD}, T}^{*}=\xi_{\mathrm{WD}, T} /\left(\epsilon_{T} c_{200}\right)$, where $\xi_{\mathrm{WD}, T}$ denotes the radial component of the tidal displacement of a mass element of the WD and $c_{200}$ is the Fourier coefficient associated with the $l=2, m=0$, and $k=0$ term in the spherical harmonic expansion of the tide-generating potential (Polfliet \& Smeyers 1990). The values of $k_{2}$ range from 0 for a point mass to 0.74 for an equilibrium sphere with uniform density. In the quasi-static tides regime, $\xi_{\mathrm{WD}, T}$ is a solution to the homogeneous secondorder differential equation (e.g., Smeyers \& Willems 2001):

$$
\begin{aligned}
\frac{d^{2} \xi_{\mathrm{WD}, T}(r)}{d r^{2}}+ & 2\left(\frac{1}{g(r)} \frac{d g(r)}{d r}+\frac{1}{r}\right) \frac{d \xi_{\mathrm{WD}, T}(r)}{d r} \\
& -\frac{l(l+1)-2}{r^{2}} \xi_{\mathrm{WD}, T}(r)=0,
\end{aligned}
$$

where $g$ denotes the local gravity. The solution to Equation (3) must remain finite at $r=0$ and $r=R_{\mathrm{WD}}$, and must satisfy the following boundary condition at the WD surface:

$$
\left(\frac{d \xi_{\mathrm{WD}, T}(r)}{d r}\right)_{R_{\mathrm{WD}}}+\frac{l-1}{R_{\mathrm{WD}}} \xi_{\mathrm{WD}, T}\left(R_{\mathrm{WD}}\right)=\epsilon_{T}(2 l+1) c_{l, 0,0}
$$

Here, $\epsilon_{T}=\left(R_{\mathrm{WD}} / a\right)^{3}\left(M_{\mathrm{NS}} / M_{\mathrm{WD}}\right)$ indicates the strength of the tidal force versus gravity at the WD's equator, $l$ is the longitudinal mode in the spherical harmonic expansion of the tide-generating potential, and $c_{l, 0,0}$ are Fourier coefficients of degree $l$. Since $c_{l, m, k}$ depend on the WD radius and the semimajor axis as $\left(R_{\mathrm{WD}} / a\right)^{l-2}$, investigations of quasi-static tides in these sources are often restricted to the dominant $l=2$ terms. Here we only consider $l=2$.

Rotation contributes to the periastron precession rate through the quadrupole distortion of the gravitational field caused by the centrifugal force (Sterne 1939) and it is given by

$$
\dot{\gamma}_{\mathrm{Rot}, \mathrm{WD}}=\frac{2 \pi}{P}\left(\frac{R_{\mathrm{WD}}}{a}\right)^{5} \frac{M_{\mathrm{NS}}+M_{\mathrm{WD}}}{M_{\mathrm{WD}}} \frac{\left(\Omega_{\mathrm{WD}} / \Omega\right)^{2}}{\left(1-e^{2}\right)^{2}} k_{2},
$$



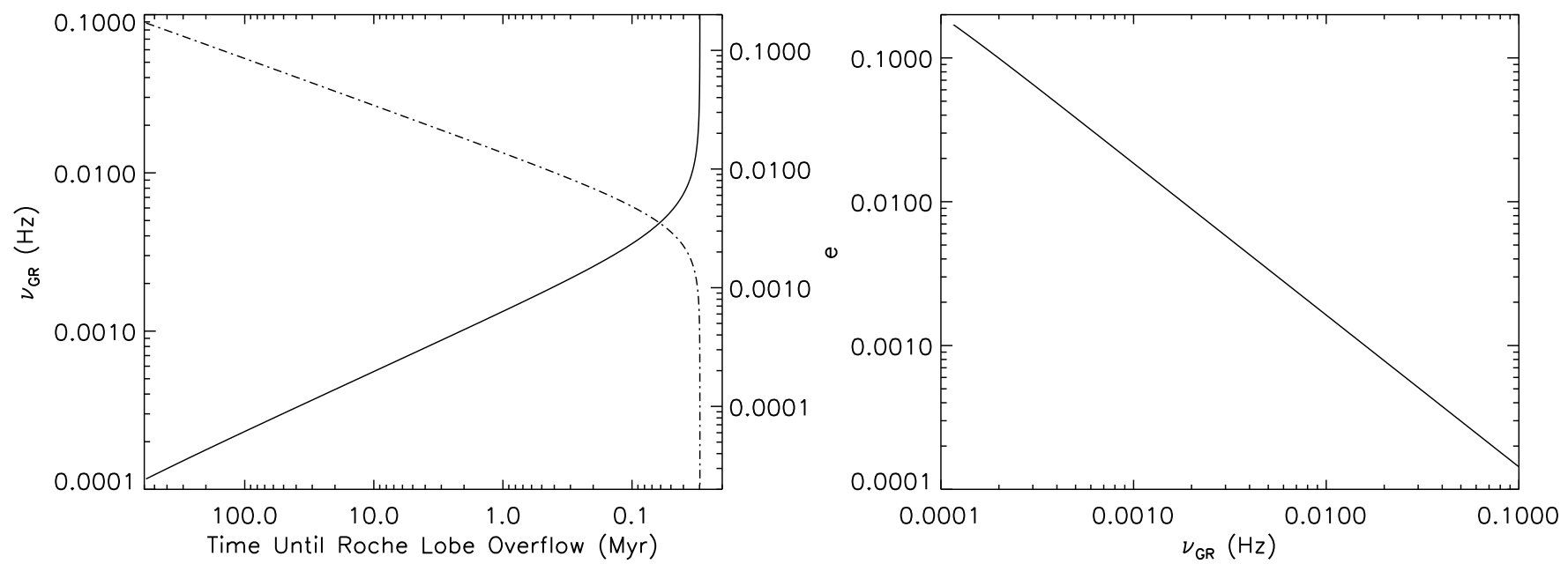

Figure 1. Left: evolution of the GW frequency ( $v_{\mathrm{GR}}$, solid line) and eccentricity ( $e$, dot-dashed line) of PSR J1141-6545 as a function of time until Roche lobe overflow. Right: evolution of eccentricity as a function of GW frequency of PSR J1141-6545.

where $\Omega=2 \pi / P$ is the mean motion. In this work, we assume synchronization at periastron for the spin of the WD component. This assumption leads to tides being dominant over rotation in driving periastron precession at any orbital frequency. The contribution due to rotation further decreases for a subsynchronous WD component. As the current observational constraints on rotation rates in single and double WD suggest that WDs are slow rotators with synchronization at periastron being an upper limit on the WD rotation rate (see the discussion in Section 3.2 of Valsecchi et al. 2011 and references therein), we can safely assume that periastron precession is primarily tidally induced at frequencies where GR is no longer important.

Finally, the GR contribution to the periastron precession rate to the leading quadrupole order is given by

$$
\dot{\gamma}_{\mathrm{GR}}=\left(\frac{30 \pi}{P}\right)^{5 / 3} \frac{3 G}{c^{2}} \frac{M_{\mathrm{NS}}+M_{\mathrm{WD}}}{a\left(1-e^{2}\right)},
$$

where $G$ is the gravitational constant and $c$ is the speed of light (Levi-Civita 1937). The total periastron precession rate is the sum of the tidal, rotational, and GR contributions.

Aside from causing periastron precession, tides and GR also induce orbital evolution. As we take tides to be quasi-static, here we only consider the evolution of orbital elements due to GR (Willems et al. 2010; but see also Burkart et al. 2013; Fuller \& Lai 2011). The time average of the rate of change of $a$ and $e$ due to GR is given by (Peters 1964)

$$
\begin{aligned}
\left\langle\frac{d a}{d t}\right\rangle= & -\frac{64}{5} \frac{G^{3} M_{\mathrm{NS}} M_{\mathrm{WD}}\left(M_{\mathrm{NS}}+M_{\mathrm{WD}}\right)}{c^{5} a^{3}\left(1-e^{2}\right)^{7 / 2}} \\
& \times\left(1+\frac{73}{24} e^{2}+\frac{37}{96} e^{4}\right) \\
\left\langle\frac{d e}{d t}\right\rangle=- & -\frac{304}{15} e \frac{G^{3} M_{\mathrm{NS}} M_{\mathrm{NS}}\left(M_{\mathrm{NS}}+M_{\mathrm{NS}}\right)}{c^{5} a^{4}\left(1-e^{2}\right)^{5 / 2}}\left(1+\frac{121}{304} e^{2}\right) .
\end{aligned}
$$

From Equations (7) and (8) it can be derived that

$$
\left\langle\frac{d a}{d e}\right\rangle=\frac{12}{19} \frac{a}{e} \frac{\left[1+(73 / 24) e^{2}+(37 / 96) e^{4}\right]}{\left(1-e^{2}\right)\left[1+(121 / 304) e^{2}\right]} .
$$

We will now make use of the above formulation to study periastron precession in eccentric NS-WD binaries. In what follows, we refer to the radius of the WD as $R$.

\section{THE OBSERVED ECCENTRIC NS-WD BINARIES: PSR J1141-6545 AND PSR B2303+46}

There are currently two known eccentric binary radio pulsars with WD companions that are believed to have formed via the mechanism described in Section 1: PSR J1141-6545 and PSR B2303+46. A summary of the properties relevant to this analysis is in Table 1. At present, neither system is a verification binary for either LISA or eLISA/NGO. However, as these systems spiral-in due to GW emission, their orbital frequencies increase. Here we examine whether PSR J1141-6545 or PSR B2303+46 still preserve an eccentricity when they evolve into the LISA sensitivity band (the sensitivity planned for LISA is from $10^{-4} \mathrm{~Hz}$ to $0.1 \mathrm{~Hz}$, henceforth "the LISA band"; that for eLISA/NGO is from $10^{-4} \mathrm{~Hz}$ to $1 \mathrm{~Hz}$ ). Moreover, if they do, we investigate the importance of tidally and rotationally induced periastron precession as they evolve throughout it.

We first consider PSR J1141-6545. We compute the evolution of its $\mathrm{GW}$ frequency $\left(v_{\mathrm{GR}}=2 v_{\mathrm{orb}}\right)$ and eccentricity as a function of time until the Roche lobe overflow using Equations (7) and (8). The results shown in Figure 1 demonstrate that this system still exhibits a small but non-zero eccentricity as it evolves throughout the LISA band. Next, we simultaneously evolve the stellar and orbital parameters to analyze the contribution of tides, rotation, and GR to periastron precession as it evolves throughout the LISA band. To model the WD component we use $1.06 M_{\odot} \mathrm{O} \backslash \mathrm{Ne} \backslash \mathrm{Mg}(\mathrm{ONeMg}) \mathrm{WD}$ models (Althaus et al. 2007; these models are described in detail in Section 4.2). At this stage we use Equations (7) and (8) and calculate $k_{2} R^{5}$ as a function of the WD age for a sequence of WD models using Equations (2)-(4). We then interpolate between the computed values.

The results of the time evolution of PSR J1141-6545 are shown in Figure 2. Here we show the individual contributions to periastron precession as a function of the GW frequency, assuming that the WD component is old $(7.6 \mathrm{Gyr})$ at present. Our results are not significantly affected by the WD evolutionary stage (see Section 4.2). The trends in Figure 2 demonstrate that, even though tides do not have a significant contribution to periastron precession in PSR J1141-6545 at present, they will be the dominant mechanism after its orbit decays to frequencies $\gtrsim 0.044 \mathrm{~Hz}$ in the next $580 \mathrm{Myr}$. 
Table 1

Properties of the Two Observed Eccentric NS-WD Binaries

\begin{tabular}{lcccccrc}
\hline \hline Name & $M_{\mathrm{NS}}$ & $M_{\mathrm{WD}}$ & $\begin{array}{c}a \\
\left(M_{\odot}\right)\end{array}$ & $\left(R_{\odot}\right)$ & $\begin{array}{c}P \\
(\mathrm{hr})\end{array}$ & $\begin{array}{c}P_{\text {spin }} \\
(\mathrm{ms})\end{array}$ & $\begin{array}{c}t_{\text {cool }} \\
(\mathrm{Myr})\end{array}$ \\
\hline PSR J1141-6545 & $1.27^{\mathrm{a}}$ & $1.02^{\mathrm{a}}$ & $1.89^{\mathrm{b}}$ & $4.744^{\mathrm{c}}$ & $393.9^{\mathrm{c}, \mathrm{d}}$ & $0.17^{\mathrm{a}}$ & $1.45^{\mathrm{c}, \mathrm{d}}$ \\
PSR B2303+46 & $1.40^{\mathrm{c}}$ & $1.24^{\mathrm{b}}$ & $31^{\mathrm{b}}$ & $296.2^{\mathrm{c}, \mathrm{d}}$ & $1066^{\mathrm{c}, \mathrm{d}}$ & $0.658^{\mathrm{c}}$ & $29.7^{\mathrm{c}, \mathrm{d}}$ \\
\hline
\end{tabular}

Notes.

a Bhat et al. (2008).

b Davies et al. (2002).

c Kalogera et al. (2004).

d van Kerkwijk et al. (1999).

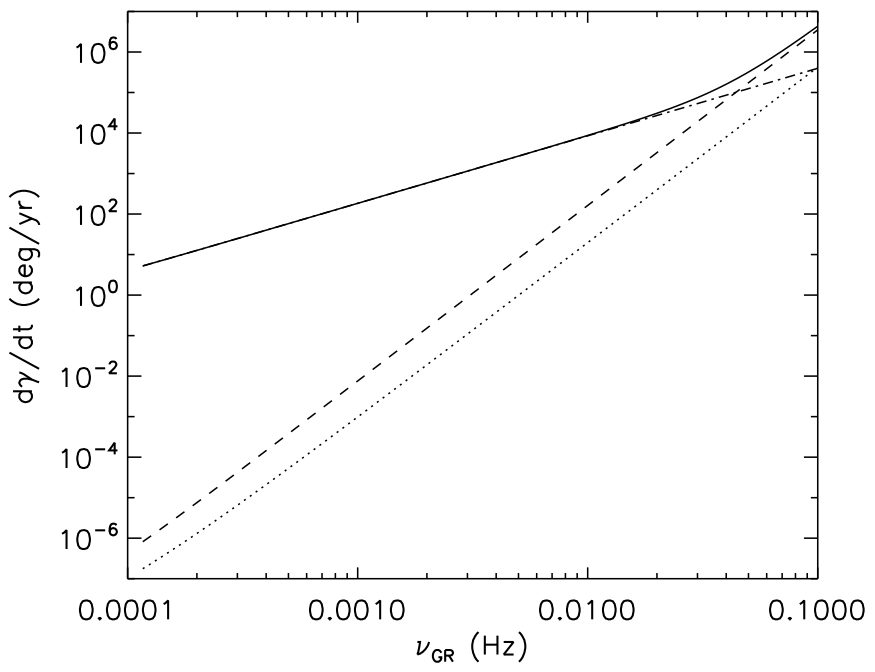

Figure 2. Periastron precession rates as a function of GW frequency for PSR J1141-6545, assuming that the WD component is old at present. The dashed line indicates the tidal contribution, the dotted line indicates the rotational contribution, and the dot-dashed line indicates the GR contribution. The solid line represents the total periastron precession rate. In a binary like PSR $\mathrm{J} 1141-6545$, the WD component will overfill its Roche lobe at $v_{\mathrm{GR}}=0.114 \mathrm{~Hz}$.

We then performed a similar analysis on PSR B2303+46. As the orbital period of this binary is much longer (see Table 1), we find that its orbital elements do not evolve significantly due to GR. As a result PSR B2303+46 will not enter the LISA band within a Hubble time.

Although the two known Galactic NS-WDs with eccentric orbits are not directly relevant to future GW observations and are not candidates for measuring periastron precession, their existence has instigated a number of studies predicting a significant Galactic population of such systems. In the rest of the study we focus on analyzing this predicted population.

\section{PERIASTRON PRECESSION FOR THE PREDICTED POPULATION OF ECCENTRIC NS-WD BINARIES}

Tutukov \& Yungelson (1993) and Nelemans et al. (2001) predict a population of galactic NS-WD binaries with an evolutionary history similar to that of PSR J1141-6545 and PSR B2303+46. Specifically, they predict $\sim 10^{4}-10^{6}$ systems for the former and $\sim 10^{6}$ systems for the latter. Tutukov \& Yungelson (1993) and Tauris \& Sennels (2000) provide detailed estimates of the expected distribution of orbital periods, eccentricities, and WD masses for such systems (see Table 2). Many of these systems will be readily detectable by LISA/eLISA/NGO and tidal effects could potentially leave an imprint on their periastron precession rate. Thus, to correctly infer the properties of these systems from periastron precession measurements, it is imperative to understand the role played by tides (Willems et al. 2008).

Apart from eccentric NS-WD binaries that form via binary evolution, Bagchi et al. (2013) recently suggested that NS-WD binaries with small but non-zero eccentricities could be formed via three-body interactions in globular clusters. We find that all such systems that are currently known have extremely small eccentricities and thus have a negligible tidal contribution to periastron precession.

Here we adopt the results of population synthesis calculations by Tauris \& Sennels (2000), as they provide the ranges in orbital periods, eccentricities, and WD masses. We analyze the predicted population through time evolution due to GR inspiral and WD cooling with a particular focus on addressing the following questions: (1) what are the binary properties of the systems that evolve into the LISA band? (2) How strong is the tidal contribution to periastron precession in these systems? (3) For the subset of systems with a significant tidal contribution, what is the mass overestimate if the sole contribution of GR is taken into account when extracting the WD mass from periastron precession rate measurements?

\subsection{Outline of the Method}

The computation of the periastron precession rates for the predicted population of eccentric NS-WD binaries proceeds as follows. First, we scan the parameter space (see Table 2) in $M_{\mathrm{WD}}$ and $\log _{10} v_{\mathrm{orb}}(\mathrm{Hz})$ in steps of $0.01 M_{\odot}$ and 0.001 , respectively. For each orbital frequency, we use Figure 4 from Tauris \& Sennels (2000) to derive the eccentricity range to be considered. For instance, systems born with long orbital periods of $\sim 1000$ days are predicted to have eccentricities of $\sim 1$. Similarly, systems born with short orbital periods of $\sim 0.003$ days are predicted to have eccentricities of $\sim 0$. In Table 3 we list the orbital period intervals considered for different eccentricities according to Figure 4 in Tauris \& Sennels (2000). Next, we scan on the derived eccentricity range in steps of 0.01 . For each combination of the above parameters, we compute the periastron precession rate by summing the contributions of GR, tides, and rotation.

Once the total periastron precession rate has been calculated, we extract the mass of the WD component assuming that only GR contributes to the precession, thus computing the error in the mass inferred for each combination of WD mass, orbital frequency, and eccentricity.

\subsection{Simplifying the Tidal and Rotational Contributions to Periastron Precession: $k_{2} R^{5}$ Versus $M_{W D}$}

Following Valsecchi et al. (2011), we investigate whether the tidal and rotational contributions to periastron precession 

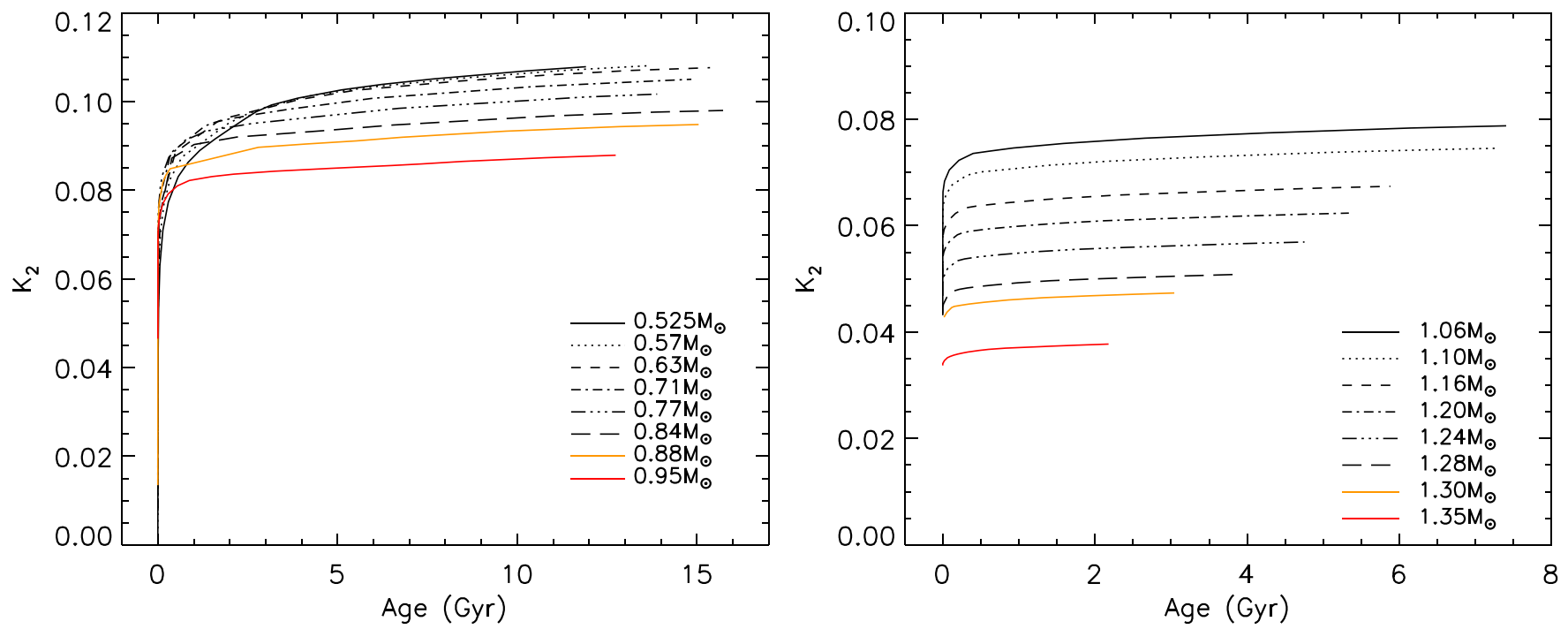

Figure 3. $k_{2}$ as a function of age for $\mathrm{CO}$ (left) and $\mathrm{ONeMg}$ (right) WDs.

(A color version of this figure is available in the online journal.)

Table 2

Parameter Space Predicted by Population Synthesis

\begin{tabular}{lcccccc}
\hline \hline Reference & Type of WD & Galactic Population & $\begin{array}{c}M_{\mathrm{NS}} \\
\left(M_{\odot}\right)\end{array}$ & $\begin{array}{c}M_{\mathrm{WD}} \\
\left(M_{\odot}\right)\end{array}$ & $P_{\text {orb }}$ & $e$ \\
\hline Tutukov \& Yungelson (1993) & & & & $0.01-1000$ & $0.2-0.6,0.8-1.0$ \\
Tutukov \& Yungelson (1993) & ONe & $0.99 \times 10^{4}-0.31 \times 10^{6}$ & 1.40 & $1.12-1.40$ & $0.1-1000$ & $0.2-1.0$ \\
Tauris \& Sennels (2000) & Not specified & Not specified & 1.30 & $0.6-1.4$ & $0.003-1000$ & $0.0-1.0$ \\
\hline
\end{tabular}

Table 3

Bounds on Orbital Period Correlation to Eccentricity

\begin{tabular}{lllccc}
\hline \hline Bound & $\begin{array}{c}e=0.2 \\
(\text { days })\end{array}$ & $\begin{array}{c}e=0.4 \\
(\text { days })\end{array}$ & $\begin{array}{c}e=0.6 \\
(\text { days })\end{array}$ & $\begin{array}{c}e=0.8 \\
(\text { days })\end{array}$ & $\begin{array}{c}e=1.0 \\
(\text { days })\end{array}$ \\
\hline Lower & 0.0028 & 0.0046 & 0.0062 & 0.0077 & 0.0090 \\
Upper & 6.1 & 15.5 & 26.7 & 39.3 & 1000 \\
\hline
\end{tabular}

(Equations (1) and (5), respectively) can be made dependent solely on the orbital period, eccentricity, and component masses (similar to the GR contribution in Equation (6)). Specifically, we check whether there is a one-to-one correlation between the term $k_{2} R^{5}$, entering Equations (1), and (5), and $M_{\mathrm{WD}}$. We use detailed WD models computed with the LPCODE stellar evolution code (Althaus et al. 2005). LPCODE has been used to study different problems related to the formation and evolution of WDs - see Althaus et al. (2013) and Renedo et al. (2010) for recent applications to the computation of WD cooling sequences. The input physics of the code includes the equation of state of Segretain \& Chabrier (2001) for the high-density regime-which accounts for all the important contributions for both the liquid and solid phases-complemented with an updated version of the equation of state of Magni \& Mazzitelli (1979) for the low-density regime. Radiative opacities are those of OPAL (Iglesias \& Rogers 1996), including carbonand oxygen-rich compositions, complemented with the lowtemperature opacities of Alexander \& Ferguson (1994). Conductive opacities are taken from Cassisi et al. (2007). For effective temperatures less than $10,000 \mathrm{~K}$, outer boundary conditions for the evolving models are given by detailed non-gray model atmospheres. Recently, LPCODE has been tested against other WD evolutionary codes, and uncertainties in the WD cooling ages resulting from different numerical implementations of stellar evolution equations were found to be below 2\% (Salaris et al. 2013).

For our carbon/oxygen core sequences, those with stellar masses less than $1 M_{\odot}$, the corresponding WD initial configurations are obtained from the full evolution of progenitor stars, which we computed in previous studies (Renedo et al. 2010). In those studies, progenitor stars were evolved from the zero age main sequence through the thermally pulsing and massloss phases on the asymptotic giant branch to the WD cooling phase. For our more massive WD models, those with $M>$ $1.06 M_{\odot}$, we have assumed a core composition of oxygen/neon (see Althaus et al. 2007 for details).

To calculate $k_{2}$, we use the WD models described above and Equations (2)-(4). The radius of the WD is taken from the model. In Figures 3 and 4 we plot $k_{2}$ and $k_{2} R^{5}$ as a function of the WD age, respectively, for different WD models. Figure 3 shows that, for each WD model, $k_{2}$ increases as the WD evolves. This means that the star becomes less centrally concentrated as it cools. Figure 4 shows that after $0.5 \mathrm{Gyr}$ of cooling, all WDs reach a plateau value of $k_{2} R^{5}$, which is held nearly constant for the stars' remaining lifetime. We also note that the values of $k_{2} R^{5}$ vary by less than an order of magnitude over the lifetime of any WD and the variation becomes less significant with increasing WD mass. This behavior explains the effect described in Section 3, where we find that the periastron precession rates for PSR J1 141-6545 

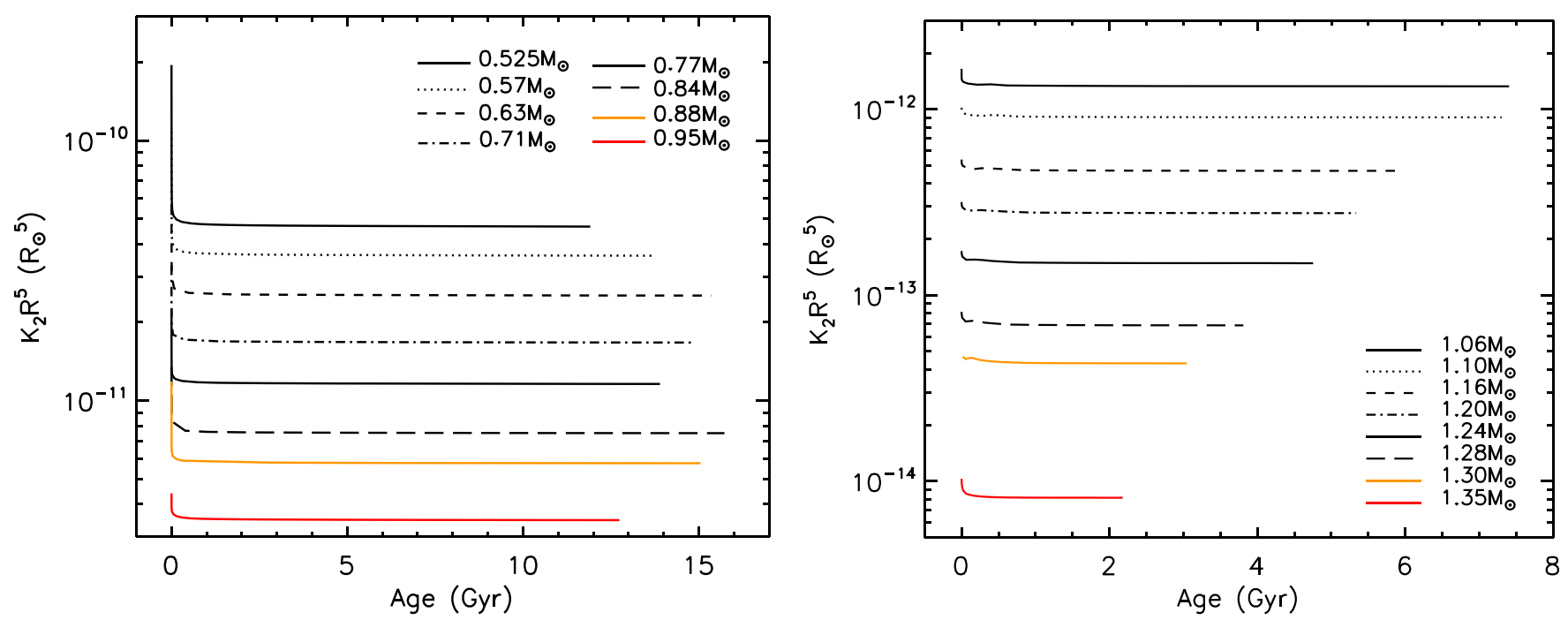

Figure 4. $k_{2} R^{5}$ as a function of age for $\mathrm{CO}$ (left) and ONeMg (right) WDs.

(A color version of this figure is available in the online journal.)

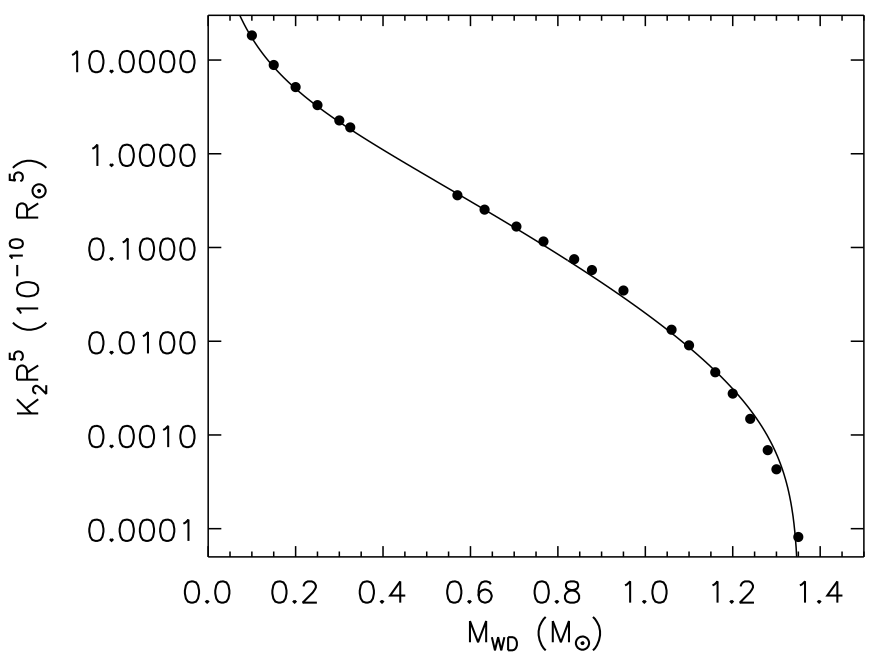

Figure 5. $k_{2} R^{5}$ as a function of $M_{\mathrm{WD}}$ for WDs $\geqslant 0.5 \mathrm{Gyr}$ old.

are not significantly affected when WDs of different ages are considered.

In Figure 5 we plot plateau values of $k_{2} R^{5}$ as a function of $M_{\mathrm{WD}}$ for the WD models described above, and those described in Valsecchi et al. (2011) and plotted in Figure 4 therein (seen here as a series of six detached points between $0.1 M_{\odot}$ and $0.35 M_{\odot}$ ). We can see that the values of $k_{2} R^{5}$ vary smoothly with $M_{\mathrm{WD}}$ for the entire set of WD models. This trend is described well by the following fitting formula:

$$
\begin{aligned}
& k_{2} R^{5}\left(10^{-10} R_{\odot}^{5}\right)=0.73 M_{\mathrm{WD}}^{-\frac{3}{2}} \exp \left(-3.6 M_{\mathrm{WD}}\right) \\
& -8.0 \exp \left(-4.8 M_{\mathrm{WD}}\right) \log \left(M_{\mathrm{WD}}\right) \sin \left(M_{\mathrm{WD}}\right),
\end{aligned}
$$

where $M_{\mathrm{WD}}$ is in solar units. This relation solves the degeneracy problem identified by Willems et al. (2008) and mentioned in Section 1 by eliminating the dependence of Equations (1) and (5) on the WD central concentration and radius. Therefore if the orbital period and eccentricity of an NS-WD binary (older than $0.5 \mathrm{Gyr}$ ) are known (from the frequency spectrum of the GW signal from these sources, for example), one can extract the mass of the WD component from periastron precession rate measurements. Because Equation (10) is only satisfied by binaries older than $0.5 \mathrm{Gyr}$, we only study the population of systems that are $\geqslant 0.5$ Gyr old in the rest of our analysis.

\subsection{Evolving the Parameter Space Forward in Time}

Given the behavior of $k_{2} R^{5}$ presented in Section 4.2, here we study the effect of tides on periastron precession for systems older than 0.5 Gyr. In particular, we consider two evolutionary stages, $0.5 \mathrm{Gyr}$ and $13.8 \mathrm{Gyr}$, corresponding to the age at which the component WD attains its plateau value of $k_{2} R^{5}$ (described by Equation (10)) and equal to a Hubble time, respectively. As the orbit of these binaries will evolve due to GR, it is important to determine how the parameter space predicted by population synthesis calculations and described in the previous sections changes after each system is evolved for 0.5 and $13.8 \mathrm{Gyr}$. Additionally, the sensitivity of LISA will place an upper (a lower) limit on the orbital frequency (orbital period).

In the top (bottom) panels of Figure 6 we plot the initial and final eccentricity ( $e_{i}$ and $e_{f}$, respectively), and the initial and final GW frequency $\left(v_{\mathrm{GR}, i}\right.$ and $v_{\mathrm{GR}, f}$, respectively) for $0.5 \mathrm{Gyr}$ (13.8 Gyr) old systems. In the left (right) panel of Figure 7, we show the final GW frequency and eccentricity for $0.5 \mathrm{Gyr}$ (13.8 Gyr) old systems. From Figure 7, we see that evolved systems with higher GW frequencies are associated with smaller eccentricities and vice versa.

From Equations (1), (5), and (6) we can see that the tidal, rotational, and GR contributions to periastron precession have different dependences on the components' masses and properties. As noted in Section 1, assuming the sole contribution of GR when extracting the component's masses from periastron precession rate measurements could lead to overestimating the mass. In this study, our method to determine the mass bias if only GR is accounted for proceeds as follows. First, we compute the periastron precession rate for each binary configuration, considering the sum of the contributions of GR, rotation, and tides. Then we extract the mass of the WD component assuming that GR is the only driver of periastron precession.

In the next section we use the evolved parameter spaces at 0.5 and 13.8 Gyr, shown in Figure 7, to investigate the importance of tides in driving periastron precession in NS-WD binaries. 

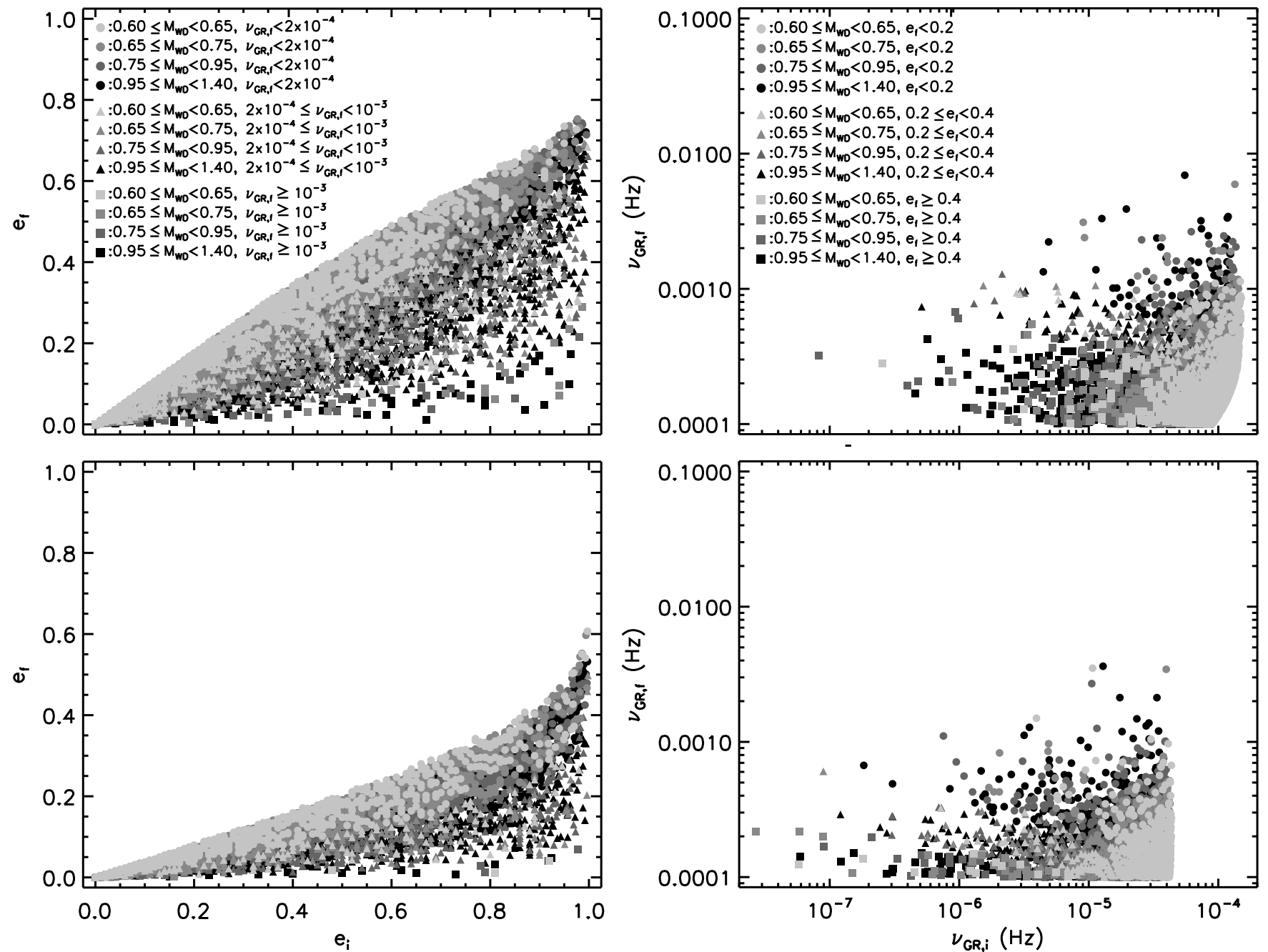

Figure 6. Final vs. initial eccentricity (left) and final vs. initial GW frequency (right) for WDs that are $0.5 \mathrm{Gyr}$ old (top) and $13.8 \mathrm{Gyr}$ old (bottom). The WD mass and frequency are in $M_{\odot}$ and $\mathrm{Hz}$, respectively. The cut-off seen in the right panels shows that systems with initial GW frequencies higher than $\sim 2 \times 10^{-4}\left(\sim 5 \times 10^{-5}\right) \mathrm{Hz}$ will evolve outside the LISA band within 0.5 (13.8) Gyr. We note that the GW frequency at which a cold $1.35 M_{\odot}\left(0.6 M_{\odot}\right)$ WD would overfill its Roche lobe is $0.48 \mathrm{~Hz}(0.034 \mathrm{~Hz})$. Since the GW frequency of a system increases with time due to GR, the upper limits on the final GW frequency seen here show that none of the systems underwent or are undergoing a phase of Roche lobe overflow.
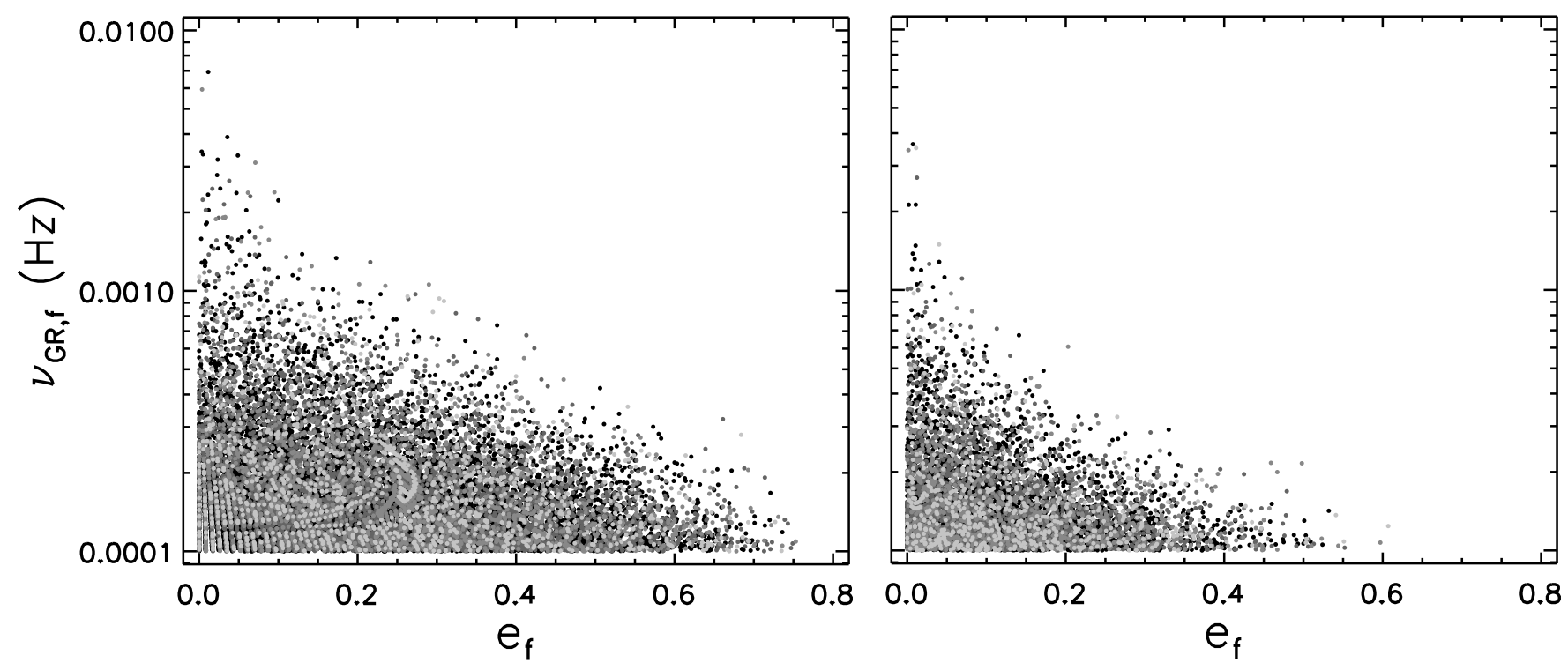

Figure 7. Final GW frequency vs. final eccentricity of systems predicted by population synthesis models that have evolved for $0.5 \mathrm{Gyr}$ (left) and $13.8 \mathrm{Gyr}$ (right). The color scheme used is the same as in Figure 6. 


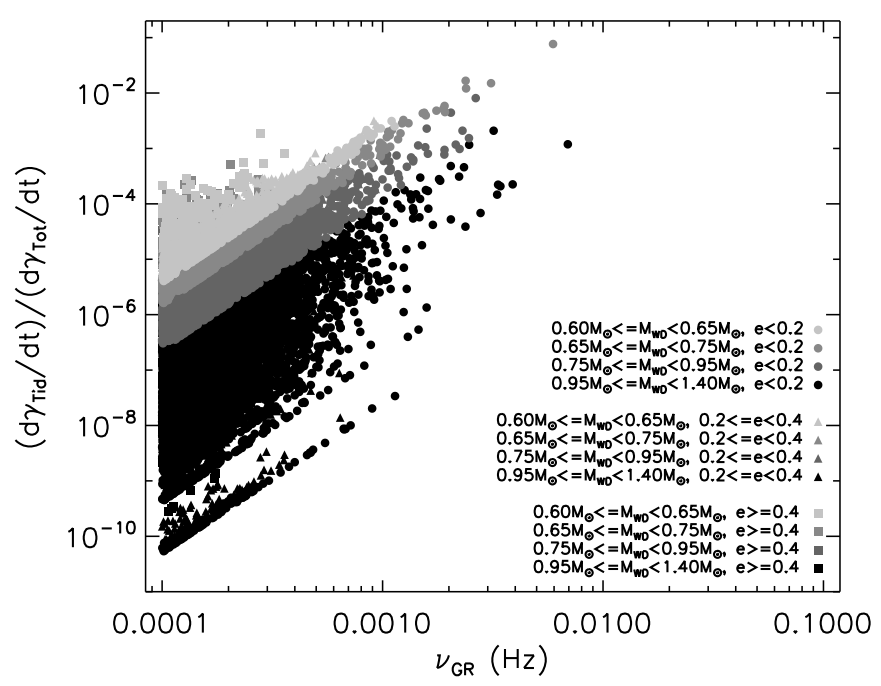

Figure 8. Ratio of the periastron precession rate due to tides to the total periastron precession rate $\left(\dot{\gamma}_{\text {Tid }} / \dot{\gamma}_{\text {tot }}\right)$ vs. GW frequency $\left(\nu_{\mathrm{GR}}\right)$ for systems that are $0.5 \mathrm{Gyr}$ old. $M_{\mathrm{WD}}$ is in units of $M_{\odot}$.

\subsection{The Periastron Precession Rates}

In Figure 8 we plot the ratio of the periastron precession rate due to tides to the total periastron precession rate $\left(\dot{\gamma}_{\text {Tid }} / \dot{\gamma}_{\text {tot }}\right)$ as a function of the $\mathrm{GW}$ frequency $\left(\nu_{\mathrm{GR}}\right)$ for $0.5 \mathrm{Gyr}$ old systems in three eccentricity ranges: $e<0.25,0.25 \leqslant e<0.5$, and $e \geqslant 0.5$. The total periastron precession rate accounts for GR, tides, and rotation. Figure 9 shows a similar calculation for $13.8 \mathrm{Gyr}$ old systems. We observe that systems with heavier WDs (darker points) have lower values of $\dot{\gamma}_{\text {Tid }} / \dot{\gamma}_{\text {tot }}$ than systems with lighter WDs (lighter points). This is because heavier WDs have smaller values of $k_{2} R^{5}$ than lighter WDs (see Section 4.2), resulting in a lower tidal contribution to the periastron precession rate. In Figure 10 we plot the total periastron precession rate as a function of $v_{\mathrm{GR}}$ for $0.5 \mathrm{Gyr}$ systems where $\dot{\gamma}_{\mathrm{Tid}} / \dot{\gamma}_{\mathrm{tot}}$ is $\sim 10^{-2}$ (top-left), $10^{-4}$ (top-right), $10^{-6}$ (bottom-left), and $10^{-8}$ (bottom-right). Figure 11 shows similar results for $13.8 \mathrm{Gyr}$ systems. As expected, the bottom-right panels in both figures are populated by heavy WDs (dark points). Similarly, the converse is seen in the top-left panels, where we can also see lighter WDs (lighter points). The value of the total periastron precession rate

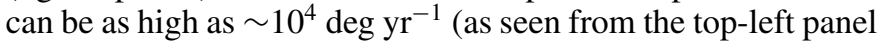
of Figure 10). Additionally, systems with a higher $\dot{\gamma}_{\text {Tid }} / \dot{\gamma}_{\text {tot }}$ also have a higher $\dot{\gamma}_{\text {tot }}$.

So far, all our calculations have assumed that the NS component has a mass of $1.3 M_{\odot}$, following population synthesis models by Tauris \& Sennels (2000). However, NSs in eccentric NS-WD binaries are observed with masses from $1.27 M_{\odot}$ to $1.40 M_{\odot}$ (see Table 1) and, when interpreting GW measurements, the actual NS mass might be unknown. Here we quantify how the uncertainty in the NS mass affects the WD mass inferred from periastron precession measurements in two ways: (1) we isolate the error introduced by the NS mass uncertainty and (2) we compute the worst-case error, arising from both neglecting tides and the NS mass uncertainty.

Figures 12 and 13 show the different errors entering the determination of the WD component mass for $0.5 \mathrm{Gyr}$ and 13.8 Gyr old systems, respectively. In gray we plot the error arising from neglecting tides and show only systems for which the relative error is $\geqslant 10 \%$. Then, for each gray data point, we calculate the total periastron precession rate. Given $\dot{\gamma}_{\text {tot }}$, we fix the orbital parameters, vary the NS mass between $1.27 M_{\odot}$ and

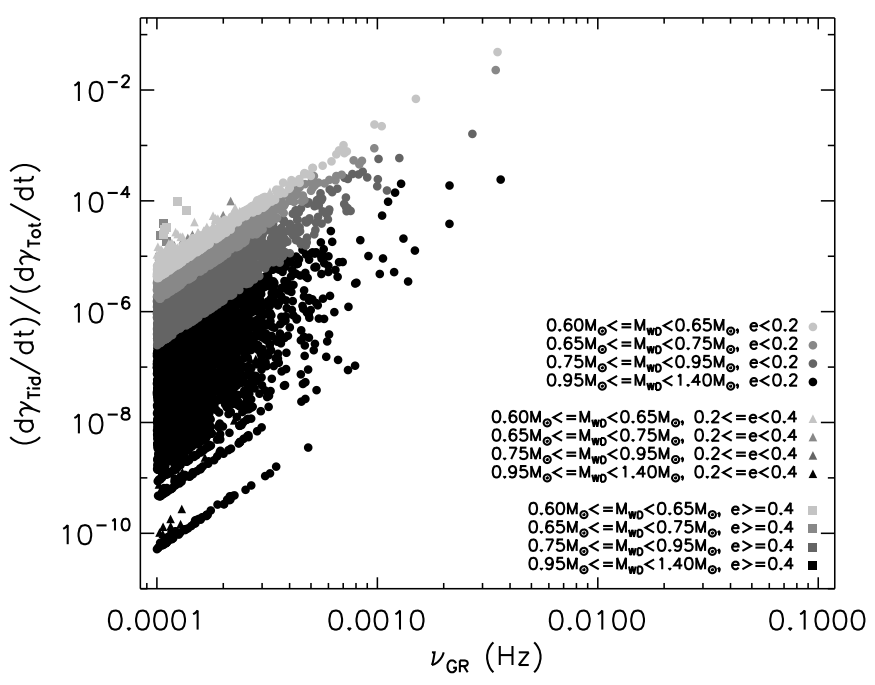

Figure 9. Ratio of the periastron precession rate due to tides to the total periastron precession rate $\left(\dot{\gamma}_{\text {Tid }} / \dot{\gamma}_{\text {tot }}\right)$ vs. GW frequency $\left(v_{\mathrm{GR}}\right)$ for systems that are $13.8 \mathrm{Gyr}$ old. $M_{\mathrm{WD}}$ is in units of $M_{\odot}$.

$1.40 M_{\odot}$, and extract two values for the WD mass, $M_{\mathrm{WD}}^{\mathrm{NS}_{1}}$ and $M_{\mathrm{WD}}^{\mathrm{NS}_{2}}$. The error due to the uncertainty in $M_{\mathrm{NS}}$ alone is then evaluated as

$$
\left(\frac{\Delta M}{M_{\text {true }}}\right)_{\text {NSmass }}=\frac{M_{\mathrm{WD}}^{\mathrm{NS}_{1}}-M_{\mathrm{WD}}^{\mathrm{NS}_{2}}}{M_{\mathrm{WD}}^{\mathrm{NS}_{\text {true }}}},
$$

where $M_{\mathrm{WD}}^{\mathrm{NS}_{\text {true }}}$ is the WD mass computed for each gray data point in Figures 12 and 13 assuming an NS mass of $1.3 M_{\odot}$. For systems that yield two solutions for $M_{\mathrm{WD}}^{\mathrm{NS}_{(1,2)}}$ (see the Appendix), the maximum value of Equation (11) is used. These uncertainties are shown in red in Figures 12 and 13.

Finally, we compute the worst-case error arising from both neglecting tides and the NS mass uncertainty. As before, we calculate $\dot{\gamma}_{\text {tot }}$ for each gray data point in Figures 12 and 13. We then set $M_{\mathrm{NS}}=1.27 M_{\odot}$ and $1.40 M_{\odot}$, and extract two WD masses, $M_{\mathrm{WD}}^{1.27}$ and $M_{\mathrm{WD}}^{1.40}$, respectively, assuming the sole contribution of GR. The worst-case error is then evaluated as

$$
\begin{aligned}
& \left(\frac{\Delta M}{M_{\text {true }}}\right)_{\text {NSmass, tides }} \\
& \quad=\operatorname{Max}\left[\frac{\left(M_{\mathrm{WD}}^{1.27}-M_{\mathrm{WD}}^{\mathrm{NS}_{\text {true }}}\right)}{M_{\mathrm{WD}}^{\mathrm{NS}}}, \frac{\left(M_{\mathrm{WD}}^{1.40}-M_{\mathrm{WD}}^{\mathrm{NS}_{\text {true }}}\right)}{M_{\mathrm{WD}}^{\mathrm{NS}_{\text {true }}}}\right] .
\end{aligned}
$$

The error thus computed is shown in blue in Figures 12 and 13.

Comparing Figure 12 (Figure 13) with Figure 8 (Figure 9) we find that systems that incur an error of $>10 \%$ due to ignoring tides have higher orbital frequencies. Since their orbits decay rapidly through this regime (for example, the orbital decay time, $t_{\mathrm{GR}}=|a / \dot{a}|$, for a circular binary with $M_{\mathrm{NS}}=1.3 M_{\odot}, M_{\mathrm{WD}}=$ $0.6 M_{\odot}$, and $v_{\mathrm{GR}}=0.01 \mathrm{~Hz}$ is $\sim 28,000 \mathrm{yr}$ ), they constitute a small fraction of the predicted population. We note that these systems should also be easier to detect owing to their relatively large GW strains.

The small fraction of systems populating Figures 12 and 13 have $\dot{\gamma}_{\text {Tid }} / \dot{\gamma}_{\text {tot }}>0.01$ and can incur errors of $>10 \%$ due to ignoring tides. However, in systems where $0.01<\dot{\gamma}_{\text {Tid }} / \dot{\gamma}_{\text {tot }} \lesssim$ 0.03 , we find that the NS mass uncertainty dominates the WD mass uncertainty, whereas, in systems where $\dot{\gamma}_{\text {Tid }} / \dot{\gamma}_{\text {tot }}>$ 0.03 , the dominant uncertainty arises due to ignoring tides. 

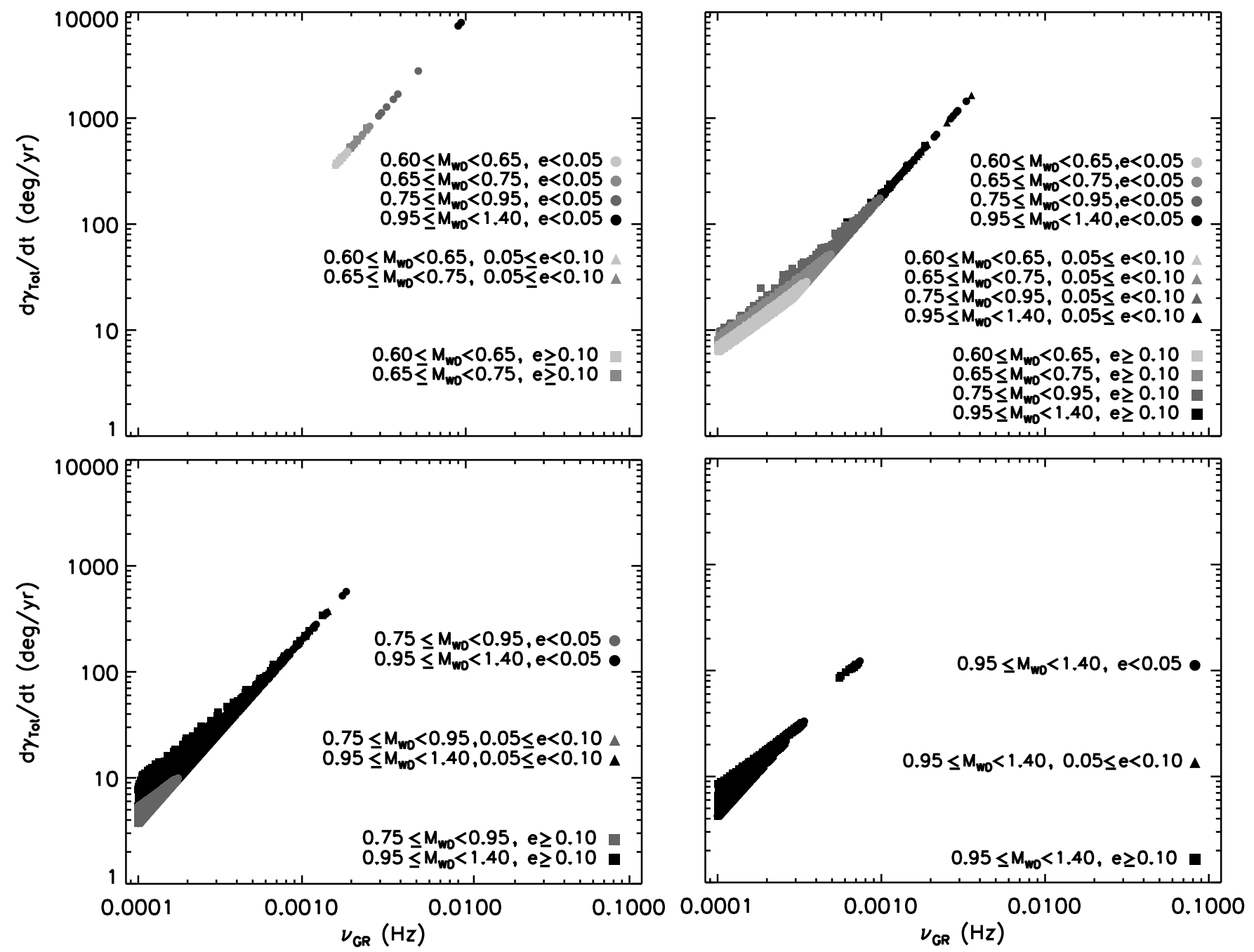

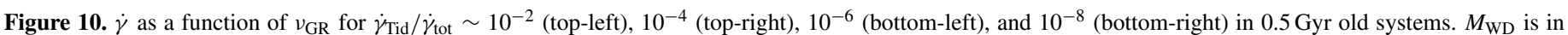
units of $M_{\odot}$.

Additionally, when $\dot{\gamma}_{\text {Tid }} / \dot{\gamma}_{\text {tot }} \gtrsim 0.1$, the error in the WD mass is $>90 \%$ and can be as high as $\sim 8000 \%$, potentially leading to a misclassification of the source, if tides are neglected. Thus, if $\dot{\gamma}_{\text {Tid }} / \dot{\gamma}_{\text {tot }} \gtrsim 0.03$, Equation (10) can be used to place constraints on the WD mass estimated from periastron precession rate measurements.

Finally, we find that the error due to the NS mass uncertainty is always $<40 \%(\lesssim 20 \%)$ in 0.5 (13.8) Gyr old systems, and decreases rapidly to $<0.1 \%$ for systems with $\dot{\gamma}_{\text {Tid }} / \dot{\gamma}_{\text {tot }} \gtrsim$ 0.1 , if tides are properly accounted for. We note that the errors become flat at small $\dot{\gamma}_{\text {Tid }} / \dot{\gamma}_{\text {tot }}$ values. As explained in the Appendix, this occurs because the range of NS masses that yields a solution for the WD mass becomes narrower as the relative tidal contribution decreases. This implies that periastron precession rate measurements can also be used to constrain the NS component mass in systems with $\dot{\gamma}_{\text {Tid }} / \dot{\gamma}_{\text {tot }}<0.1$.

\section{CONCLUSIONS}

GW emission from eccentric binaries encodes information about the rate at which the periastron of their orbit precesses. The periastron precession rate depends on the components' properties and orbital parameters. Therefore, periastron precession measurements can be used to constrain some binary properties. In this work, we focus on such precession in eccentric NS-WD binaries. These GW sources are of particular interest because their periastron precession rate can be used to place constraints on their WD component's mass if the orbital parameters are known. Additionally, since pulsar-timing measurements could yield the components' masses independent of precession effects, these systems can be used to verify the validity of our models. Here we investigate the significance of the three mechanisms driving periastron precession: tides, rotation, and GR. In particular, we focus on the tidal contribution and investigate the errors introduced in the WD mass estimated from periastron precession rate measurements, if tides are ignored.

First, we analyze tides in the two eccentric NS-WD binaries currently known: PSR J1141-6545 and PSR B2303+46. These are believed to have formed via a common envelope phase followed by a supernova event that formed the NS. For both systems, we find that tides are not the dominant driver of periastron precession at present. However, for the case of J1141-6545, we find that, as the system evolves due to GR, tides will take over as the leading mechanism in $580 \mathrm{Myr}$.

Even though we observe only two NS-WD binaries with a significant eccentricity, population synthesis studies predict the existence of a host of such systems in our galaxy, with a wide range of orbital periods and eccentricities. We analyze periastron precession in the predicted sources and find that the tidal contribution is stronger in systems having high orbital 


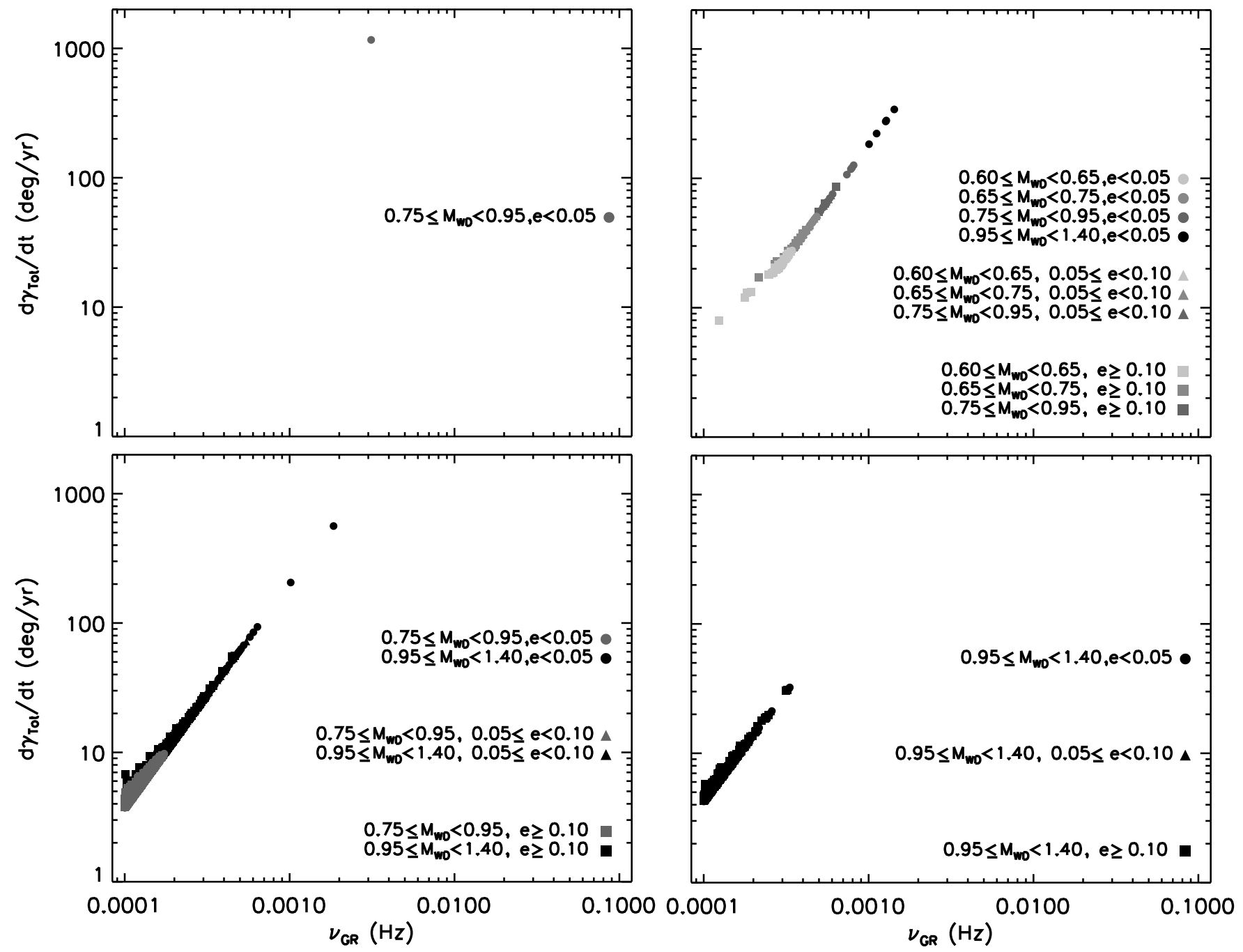

Figure 11. $\dot{\gamma}$ as a function of $\nu_{\mathrm{GR}}$ for $\dot{\gamma}_{\text {Tid }} / \dot{\gamma}_{\text {tot }} \sim 10^{-2}$ (top-left), $10^{-4}$ (top-right), $10^{-6}$ (bottom-left), and $10^{-8}$ (bottom-right) in $13.8 \mathrm{Gyr}$ old systems. $M_{\mathrm{WD}}$ is in units of $M_{\odot}$.

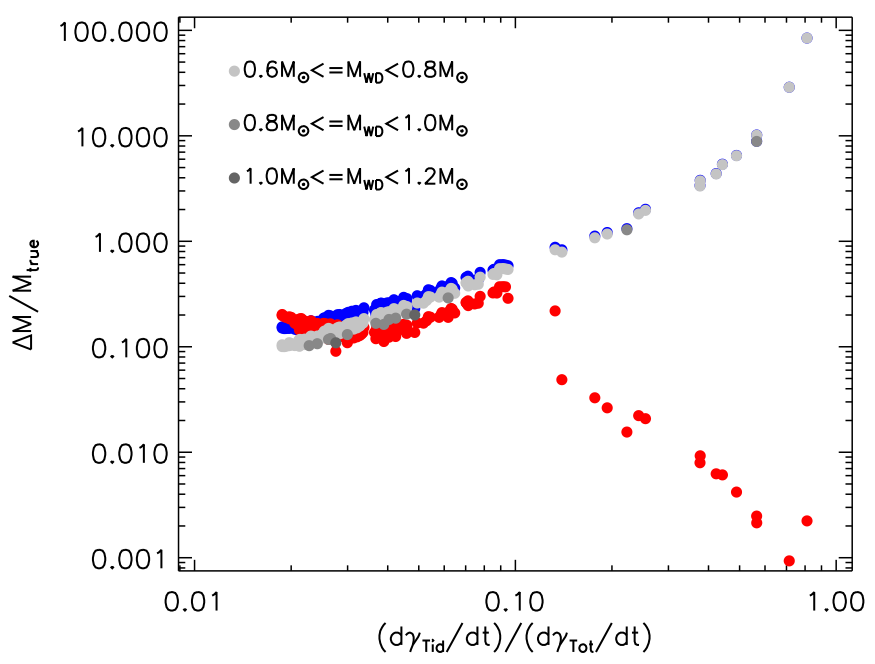

Figure 12. Relative error in the WD mass estimate as a function of $\dot{\gamma}_{\text {Tid }} / \dot{\gamma}_{\text {tot }}$ for $0.5 \mathrm{Gyr}$ old systems due to neglecting tides (gray), NS mass uncertainty (red), and both neglecting tides and NS mass uncertainty (blue).

(A color version of this figure is available in the online journal.)

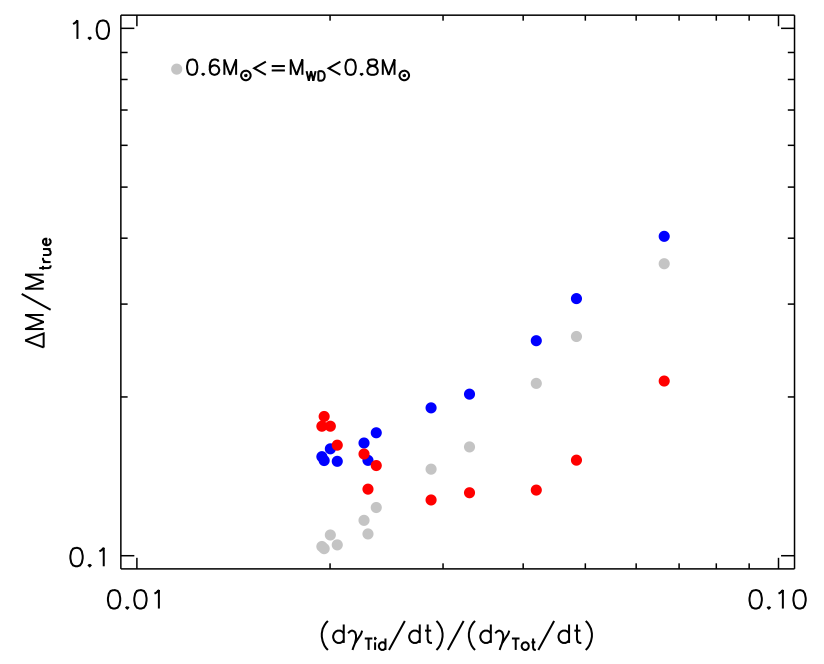

Figure 13. Relative error in the WD mass estimate as a function of $\dot{\gamma}_{\text {Tid }} / \dot{\gamma}_{\text {tot }}$ for 13.8 Gyr old systems due to neglecting tides (gray), NS mass uncertainty (red), and both neglecting tides and NS mass uncertainty (blue).

(A color version of this figure is available in the online journal.) 


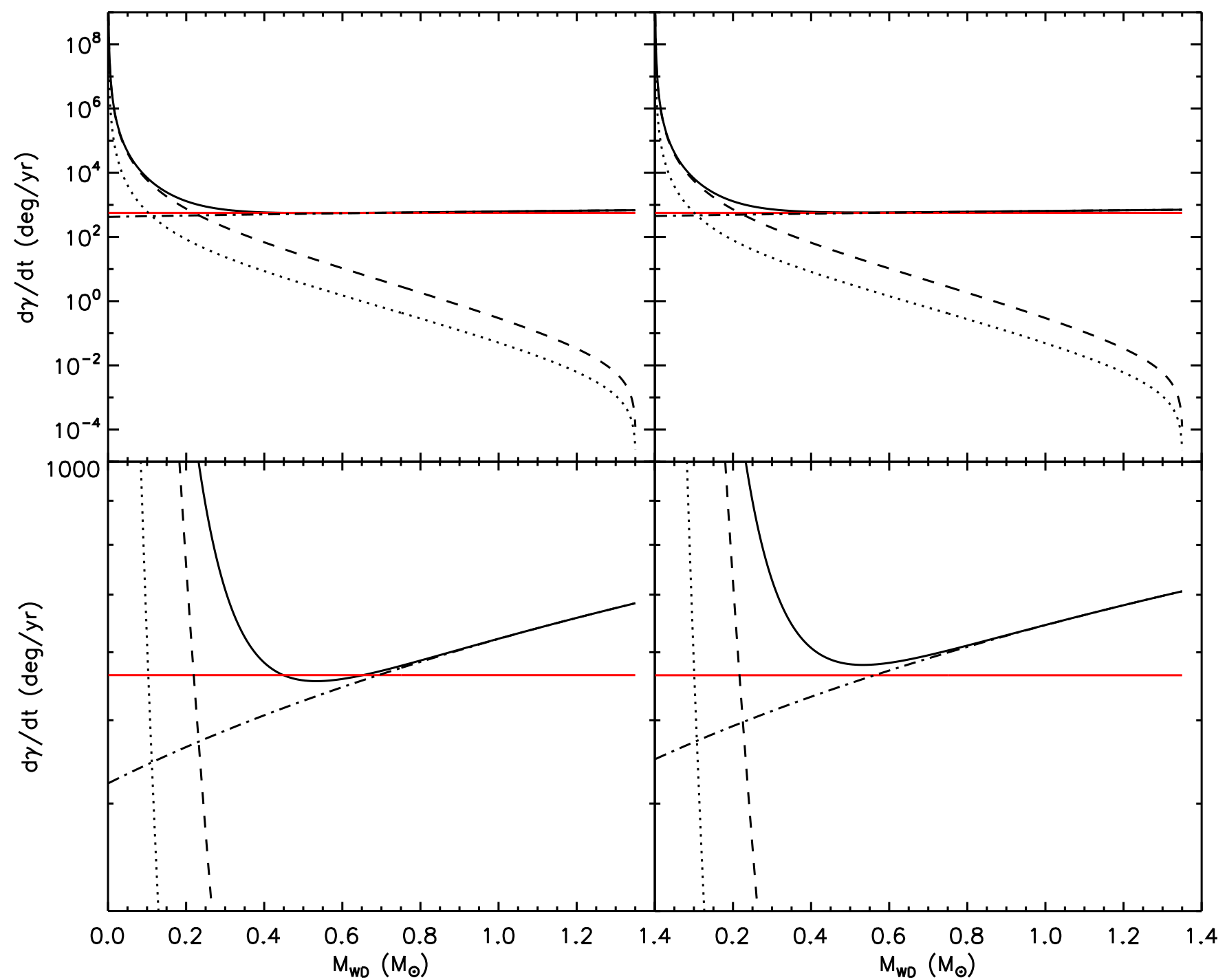

Figure 14. System A: $\dot{\gamma}$ as a function of $M_{\mathrm{WD}}$ for $M_{\mathrm{NS}}$ changed to $1.27 M_{\odot}$ (left) and $1.40 M_{\odot}$ (right). The dashed line indicates the tidal contribution, the dotted line indicates the rotational contribution, and the dot-dashed line indicates the GR contribution. The solid line represents the total periastron precession rate. The solid red line is the total periastron precession rate we want to match by changing the WD mass. The bottom panels show a blow-up of the regions where the solid red and black lines are comparable in magnitude.

(A color version of this figure is available in the online journal.)

frequencies, high eccentricities, and low WD masses (i.e., larger radii). Furthermore, the tidal contribution grows more rapidly with increasing orbital frequency than eccentricity. In the majority of the systems, the relative tidal contribution is small $(<1 \%)$ and the error in the WD mass inferred would be $<10 \%$ if tides are ignored. In systems where the relative tidal contribution is between $1 \%$ and $3 \%$, the NS mass uncertainty dominates the errors in the inferred WD mass. In systems where the relative tidal contribution is between $3 \%$ and $10 \%$, the errors arising due to ignoring tides dominate. However, in this regime the NS mass uncertainty limits the accuracy with which the WD mass can be inferred. Regardless, the error arising solely due to the NS mass uncertainty is always less than $40 \%$ ( $20 \%$ for the oldest systems). Finally, in systems where the relative tidal contribution is $>10 \%$, tides play a significant role and, if neglected, the error in the WD mass inferred from periastron precession measurements is $>90 \%$ and could be as high as $\sim 8000 \%$. Clearly, in these extreme cases, neglecting tides would lead to a misclassification of the source. However, since systems with relative tidal contribution $>1 \%$ have higher orbital frequencies, they decay rapidly, thereby constituting only a small fraction of the predicted population.

Accounting for the tidal (and rotational) contributions to periastron precession introduces the periastron precession constant $k_{2}$ and the WD radius $R$, as $k_{2} R^{5}$, which becomes an additional parameter. We show that $k_{2} R^{5}$ is a smooth function of the WD mass for most of the WD lifetime (starting at $0.5 \mathrm{Gyr}$ of its cooling age) and derive a relation between $k_{2} R^{5}$ and the WD mass. This relation can be used to simplify the equations governing the total periastron precession rate, facilitating a more accurate extraction of the WD mass from periastron precession measurements.

We conclude that while accounting for tides when interpreting periastron precession rates to determine the WD component's mass in eccentric NS-WD binaries is not necessary in most cases, tidal precession can be accounted for by using the relationship between $k_{2} R^{5}$ and WD mass presented here, thereby improving constraints on inferred WD mass in some cases. Gravitational waves emerging from such sources will provide a new astrophysical laboratory to test the reliability of our current 


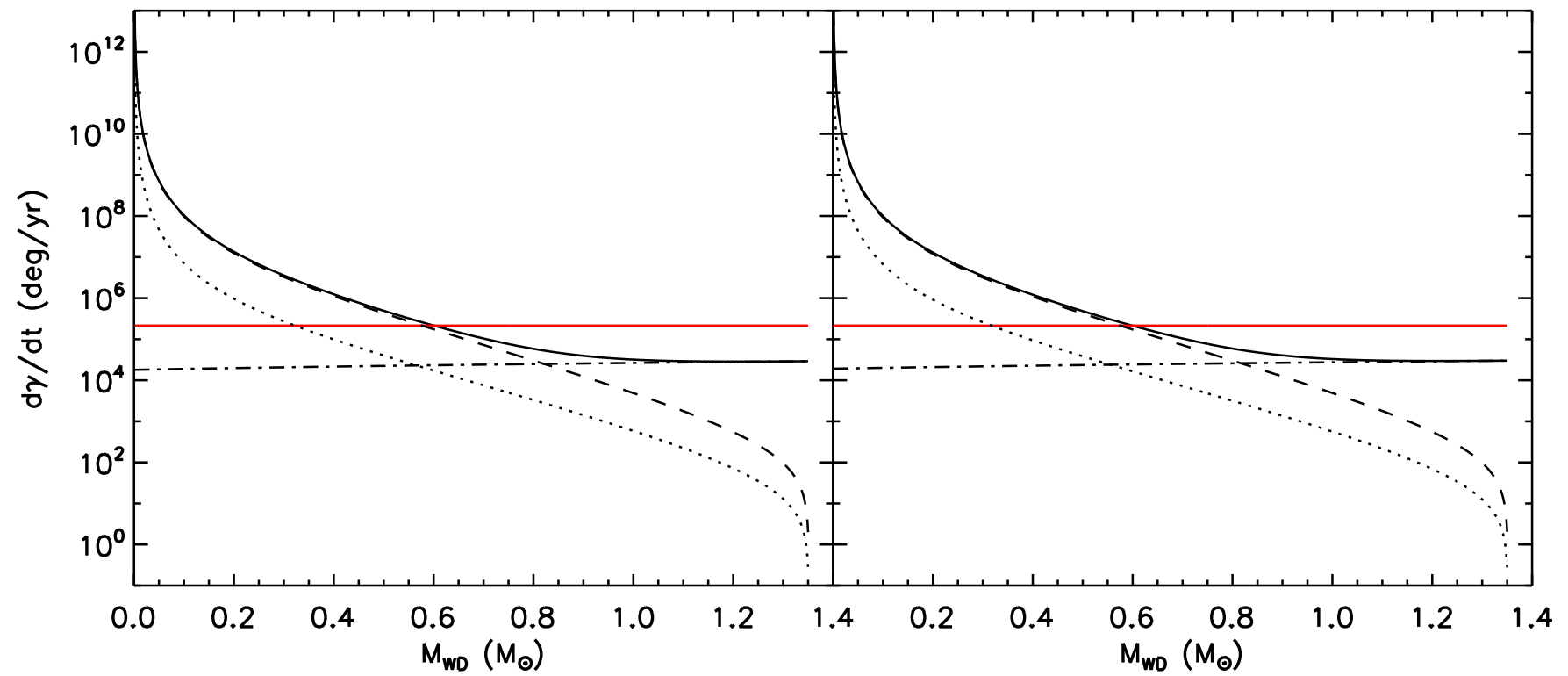

Figure 15. System B: $\dot{\gamma}$ as a function of $M_{\mathrm{WD}}$ for $M_{\mathrm{NS}}$ changed to $1.27 M_{\odot}$ (left) and $1.40 M_{\odot}$ (right). The dashed line indicates the tidal contribution, the dotted line indicates the rotational contribution, and the dot-dashed line indicates the GR contribution. The solid line represents the total periastron precession rate. The solid red line is the total periastron precession rate we want to match by changing the WD mass.

(A color version of this figure is available in the online journal.)

Table 4

Properties of Systems A and B

\begin{tabular}{lccccccc}
\hline \hline System & $\begin{array}{c}M_{\mathrm{NS}} \\
\left(M_{\odot}\right)\end{array}$ & $\begin{array}{c}M_{\mathrm{WD}} \\
\left(M_{\odot}\right)\end{array}$ & $e$ & $v_{\text {orb }}$ & $\begin{array}{c}\dot{\gamma}_{\text {tot }} \\
\left(\mathrm{deg} \mathrm{yr}^{-1}\right)\end{array}$ & $\dot{\gamma}_{\text {Tid }} / \dot{\gamma}_{\text {tot }}$ & $\Delta M / M_{\text {true }}$ \\
\hline A & 1.30 & 0.60 & $1.00 \times 10^{-1}$ & $1.03638 \times 10^{-3}$ & $5.643 \times 10^{2}$ & 0.018744 & 0.10435 \\
B & 1.30 & 0.60 & $1.43 \times 10^{-3}$ & $9.88927 \times 10^{-3}$ & $2.147 \times 10^{5}$ & 0.811203 & 84.4638 \\
\hline
\end{tabular}

understanding of the physics governing these systems, and perhaps even unravel elements of the engines fueling compact object physics that remain shrouded by conventional observation techniques in the electromagnetic spectrum.

We thank Bart Willems for useful discussions, and the anonymous referee for his/her positive and constructive review. Vicky Kalogera is grateful for support through a Simons Foundation Fellowship in Theoretical Physics and for the hospitality of the Aspen Center for Physics.

\section{APPENDIX}

\section{CONSTRAINING THE COMPONENT MASSES FROM PERIASTRON PRECESSION RATES}

Here we explain the procedure proposed in Section 4.4 to compute the error in the extracted WD mass arising from the NS mass uncertainty alone. First, for a given system, the total periastron precession rate is computed using Equations (1), (5), and (6), assuming an NS mass of $1.3 M_{\odot}$. Next, we vary the NS mass between $1.27 M_{\odot}$ and $1.40 M_{\odot}$ while keeping the orbital parameters fixed and, for each NS mass, we search for values of $M_{\mathrm{WD}}$ that yield the same $\dot{\gamma}_{\text {tot }}$. The computation of these values is not straightforward and warrants a discussion of the behavior of $\dot{\gamma}_{\mathrm{tot}}$ as a function of $M_{\mathrm{WD}}$. Since this behavior changes significantly as $\dot{\gamma}_{\text {Tid }} / \dot{\gamma}_{\text {tot }}$ increases, we focus on the two systems at the extreme ends of Figure 12, that is, the ones with the smallest (A) and largest (B) value of $\dot{\gamma}_{\text {Tid }} / \dot{\gamma}_{\text {tot }}$. Their properties are outlined in Table 4.

\section{A.1. System A}

This system has the smallest value of $\dot{\gamma}_{\text {Tid }} / \dot{\gamma}_{\text {tot }}$ that yields an error $>10 \%$ in the WD mass estimated from periastron precession rate measurements due to ignoring tides. In Figure 14 we plot the individual and total contributions to the periastron precession rate as a function of $M_{\mathrm{WD}}$ on changing the NS mass in this system to $1.27 M_{\odot}$ (left) and $1.40 M_{\odot}$ (right) in black. The solid red line shows the value of the total periastron precession rate we want to match (see Table 4). We search for the values of $M_{\mathrm{WD}}$ where the solid black and red curves intersect. We find that the tidal contribution to periastron precession is stronger for smaller values of $M_{\mathrm{WD}}$. The reverse is seen for the GR contribution. Therefore, the total periastron precession rate first decreases and then increases as a function of $M_{\mathrm{WD}}$. The bottom panels of Figure 14 show a blow-up of the region where the two curves are comparable in magnitude for each $M_{\mathrm{NS}}$. We find that the total periastron precession rate increases on increasing $M_{\mathrm{NS}}$. This results in two solutions for $M_{\mathrm{WD}}$ when $M_{\mathrm{NS}}$ is set to 1.27 $M_{\odot}$, and no solutions when set to $1.40 M_{\odot}$. This behavior is typical for all systems where $\dot{\gamma}_{\text {Tid }} / \dot{\gamma}_{\text {tot }} \lesssim 0.1$. Therefore, to find a solution to Equation (11) for a system like System A, $M_{\mathrm{NS}}$ needs to be decreased from $1.40 M_{\odot}$ until the two curves intersect. Additionally, this implies that $\dot{\gamma}_{\text {tot }}$ could also be used to constrain the NS mass in these systems, since only a subset of the values of $M_{\mathrm{NS}}$ yield a solution for $M_{\mathrm{WD}}$ for a given value of $\dot{\gamma}_{\text {tot }}$.

\section{A.2. System $B$}

In Figure 15, we show results for System B, which are similar to those described above for System A. Since this system has 
a strong tidal contribution, $\dot{\gamma}_{\text {tot }}$ decreases as a function of $M_{\mathrm{WD}}$ for most of the range in $M_{\mathrm{WD}}$ considered. This results in a single solution for $M_{\mathrm{WD}}$ for both values of $M_{\mathrm{NS}}$. As before, we find that the curve for $\dot{\gamma}_{\text {tot }}$ shifts up as $M_{\mathrm{NS}}$ is increased, but in this case the roots for each NS mass are nearly the same. A comparison of Figures 14 and 15 shows that as the relative tidal contribution increases, the curve for $\dot{\gamma}_{\text {tot }}$ spreads out. Thus, even though a second solution for $M_{\mathrm{WD}}$ could theoretically exist for systems with $\dot{\gamma}_{\text {Tid }} / \dot{\gamma}_{\text {tot }}>0.1$, its value would be greater than the Chandrasekhar limit.

\section{REFERENCES}

Amaro-Seoane, P., Aoudia, S., Babak, S., et al. 2012, CQGra, 29, 124016

Alexander, D. R., \& Ferguson, J. W. 1994, ApJ, 437, 879

Althaus, L. G., García-Berro, E., Isern, J., Córsico, A. H., \& Rohrmann, R. D. 2007, A\&A, 465, 249

Althaus, L. G., Miller Bertolami, M. M., \& Córsico, A. H. 2013, A\&A, 557, A19

Althaus, L. G., Serenelli, A. M., Panei, J. A., et al. 2005, A\&A, 435, 631

Bagchi, M., Lorimer, D. R., \& Wolfe, S. 2013, MNRAS, 432, 1303

Bhat, N. D. R., Bailes, M., \& Verbiest, J. P. W. 2008, PhRvD, 77, 124017

Brown, G. E., Lee, C. -H., Portegies Zwart, S. F., \& Bethe, H. A. 2001, ApJ, 547,345

Burkart, J., Quataert, E., \& Arras, P. 2013, arXiv:1312.4966

Cassisi, S., Potekhin, A. Y., Pietrinferni, A., Catelan, M., \& Salaris, M. 2007, ApJ, 661, 1094

Church, R. P., Bush, S. J., Tout, C. A., \& Davies, M. B. 2006, MNRAS, 372,715

Cowling, T. G. 1938, MNRAS, 98, 734

Danzmann, K., \& The LISA Study Team. 1996, CQGra, 13, 247
Davies, M. B., Ritter, H., \& King, A. 2002, MNRAS, 335, 369

Fuller, J., \& Lai, D. 2011, MNRAS, 412, 1331

Hughes, S. A. 2006, in AIP Conf. Proc. 873, Laser Interferometer Space Antenna: 6th International LISA Symposium, ed. S. M. Merkovitz \& J. C. Livas (Melville, NY: AIP), 13

Iglesias, C. A., \& Rogers, F. J. 1996, ApJ, 464, 943

Kalogera, V., Kim, C., Lorimer, D. R., Ihm, M., \& Belczynski, K. 2004, in ASP Conf. Ser. 328, Binary Radio Pulsars, ed. F. A. Rasio \& I. H. Stairs (San Francisco, CA: ASP), 261

Kaspi, V. M., Lyne, A. G., Manchester, R. N., et al. 2000, ApJ, 543, 321

Levi-Civita, T. 1937, AmJM, 59, 225

Magni, G., \& Mazzitelli, I. 1979, A\&A, 72, 134

Nelemans, G., Yungelson, L. R., \& Portegies Zwart, S. F. 2001, A\&A, 375, 890

Peters, P. C. 1964, PhRvB, 136, 1224

Polfliet, R., \& Smeyers, P. 1990, A\&A, 237, 110

Portgies Zwart, S. F., \& Yungelson, L. R. 1999, MNRAS, 309, 26

Renedo, I., Althaus, L. G., Miller Bertolami, M. M., et al. 2010, ApJ, 717, 183

Salaris, M., Althaus, L. G., \& García-Berro, E. 2013, A\&A, 555, A96

Segretain, L., \& Chabrier, G. 1993, A\&A, 271, L13

Seto, N. 2001, PhRvL, 87, 251101

Smeyers, P., \& Willems, B. 2001, A\&A, 373, 173

Sterne, T. E. 1939, MNRAS, 99, 451

Tauris, T. M., \& Sennels, T. 2000, A\&A, 355, 236

Tutukov, A. V., \& Yungelson, L. R. 1993, ARep, 37, 411

Valsecchi, F., Farr, W. M., Willems, B., Deloye, C. J., \& Kalogera, V. 2011, ApJ, 745,137

van Kerkwijk, M. H., \& Kulkarni, S. R. 1999, ApJL, 516, L25

Willems, B., \& Claret, A. 2002, A\&A, 382, 1009

Willems, B., \& Claret, A. 2005, in ASP Conf. Ser. 333, Dynamic Tides and the Evolution of Stars in Close Binaries, ed. A. Claret, A. Giménez, \& J.-P. Zahn (San Francisco, CA: ASP), 52

Willems, B., Deloye, C. J., \& Kalogera, V. 2010, ApJ, 713, 239

Willems, B., Kalogera, V., Vecchio, A., et al. 2007, ApJL, 665, L59

Willems, B., Vecchio, A., \& Kalogera, V. 2008, PhRvL, 100, 041102 